\title{
Challenges in the functional diagnosis of thyroid nodules before surgery for TSH-producing pituitary adenoma
}

\author{
Keita Tatsushima1, Akira Takeshita1, Shuji Fukata², Noriaki Fukuhara1, \\ Mitsuo Yamaguchi-Okada1, Hiroshi Nishioka1 and Yasuhiro Takeuchi1 \\ 'Endocrine Centre, Toranomon Hospital, Minato-ku, Tokyo, Japan and 2Kuma Hospital, Kobe, Japan
}

\author{
Correspondence \\ should be addressed \\ to K Tatsushima \\ Email \\ tatsushima_kyu@icloud.com
}

\section{Summary}

A 50-year-old woman with thyroid-stimulating hormone (TSH)-producing pituitary adenoma (TSHoma) was diagnosed due to symptoms of thyrotoxicosis. Preoperatively, she showed thyrotoxicosis with the syndrome of inappropriate secretion of TSH (SITSH) and had a $5 \mathrm{~cm}$ nodule in her thyroid gland. Octreotide was administered preoperatively, which helped lower her serum TSH level but not her thyroid hormone level. These findings were atypical for a patient with TSHoma. The TSHoma was completely resected, and the TSH level dropped below the sensitivity limit shortly after surgery. Interestingly, however, thyroid hormone levels remained high. A clear clue to the aetiology was provided by consecutive thyroid scintigraphy. Although preoperative thyroid scintigraphy did not show a hot nodule and the mass was thought to be a non-functional thyroid nodule, the nodule was found to be hot in the postoperative phase of TSH suppression. By focusing on the atypical postoperative course of the TSHoma, we were able to conclude that this was a case of TSHoma combined with an autonomously functioning thyroid nodule (AFTN).

\section{Learning points:}

- The diagnosis of autonomously functioning thyroid nodules (AFTNS) depends on suppressed serum TSH levels.

- If thyroid hormones are resistant to somatostatin analogue therapy or surgery for TSHoma, complications of AFTN as well as destructive thyroiditis need to be considered.

- It is important to revisit the basics when facing diagnostic difficulties and not to give up on understanding the pathology.

\section{Background}

Thyroid-stimulating hormone (TSH)-producing pituitary adenoma (TSHoma) is a relatively rare functional tumour that accounts for $0.5-3 \%$ of pituitary adenomas (1). The disease may be associated with symptoms of thyrotoxicosis, such as tachycardia and hyperhidrosis, as well as headache and visual field disturbances due to the mass effect of the tumour. Eighty-five per cent of patients with TSHoma present with diffuse goitre (2), often associated with nodules in the thyroid gland. However, the function of thyroid nodules is not well described.
On the other hand, autonomously functioning thyroid nodules (AFTNs) are caused by gain-of-function somatic mutations of the TSH receptor (TSHR) or $\alpha$ subunit of GTP-binding protein (GNAS) that lead to thyrotoxicosis independent of TSH (3). AFTNs are more common in areas with iodine deficiencies and less common in areas with excessive iodine intake, including Japan. AFTNs usually present with findings of primary hyperthyroidism and are diagnosed by thyroid scintigraphy, which shows thyroid lesions as hot nodules. 
The present case is a rare combination of TSHoma and an AFTN. AFTN was not diagnosed preoperatively in the presence of TSHoma, and the atypical preoperative and postoperative course of the patient revealed the diagnosis of AFTN. Herein, we report this educational case.

\section{Case presentation}

A 50-year-old woman visited her previous doctor with complaints of palpitations, neck swelling and excessive sweating. These symptoms appeared 5 years earlier and gradually worsened. She had no notable medical or family history. She did not have any history of smoking or drinking. Her pulse was 92 b.p.m., and a nodule was palpable on her thyroid gland. There was no weight loss.

\section{Investigation}

Thyroid function tests performed by the previous doctor showed high FT3 and FT4 levels and inadequate suppression of TSH on multiple occasions, which was a finding of syndrome of inappropriate secretion of TSH (SITSH) (Table 1). Moreover, both TRAb and TSAb were negative, while TPOAb and TgAb were both positive. There were no other abnormalities in the other pituitary hormone levels, general blood samples, or the TR $\beta 1$ gene. Pituitary MRI revealed a 5-mm tumour with poor enhancement on contrast-enhanced T1-weighted images (Fig. 1). The TRH loading test resulted in a low response for TSH (1.093-1.771 $\mathrm{mIU} / \mathrm{L})$. Based on these data, the patient was diagnosed as TSHoma and came to our hospital for pituitary surgery.
Thyroid ultrasound demonstrated a nodule greater than $5 \mathrm{~cm}$ on the caudal side of the thyroid gland, and the thyroid parenchyma suggested Hashimoto's disease (Fig. $2 \mathrm{~A}$ and $\mathrm{B}$ ). On ${ }^{99 \mathrm{~m}_{\mathrm{TCO}}}$ scintigraphy, the accumulation in the nodule was equivalent to that in the thyroid parenchyma (Fig. 2C).

\section{Treatment}

Since the patient showed thyrotoxicosis upon admission, we prescribed potassium iodide $100 \mathrm{mg}$ daily and subcutaneous injections of octreotide $300 \mu \mathrm{g}$ per day for 3 days to normalise her thyroid function before surgery. The TSH decreased to substandard values, but interestingly, serum FT3 and FT4 levels remained unchanged. With extreme care, the surgery for TSHoma was performed as planned, and the tumour was completely removed without complications. The resected tumour was found to be a TSH-, PRL-, PIT-1-, and somatostatin receptor $2 \mathrm{~A}$ (SSTR2A)-positive adenoma by immunostaining, whereas it was GH-negative. The diagnosis of TSHoma was confirmed based on these results (Fig. 3).

\section{Outcome and follow-up}

The serum TSH level decreased to under the sensitivity limit immediately after surgery, whereas high serum FT3 and FT4 values persisted. It was an atypical course, as serum TSH levels usually quickly fall to below the sensitivity limit, and FT3 and FT4 typically fall to the

Table 1 Pituitary and thyroid hormone profile.

\begin{tabular}{l}
\hline Hormone \\
\hline TSH $(\mathrm{mlU} / \mathrm{L})$ \\
fT3 $(\mathrm{pg} / \mathrm{mL})$ \\
fT4 $(\mathrm{ng} / \mathrm{dL})$ \\
Thyrogloblin $(\mathrm{ng} / \mathrm{mL})$ \\
GH $(\mathrm{ng} / \mathrm{mL})$ \\
IGF-1 $(\mathrm{ng} / \mathrm{mL})$ \\
Prolactin $(\mathrm{ng} / \mathrm{mL})$ \\
ACTH $(\mathrm{pg} / \mathrm{mL})$ \\
Cortisol $(\mu \mathrm{rg} / \mathrm{dL})$ \\
LH $(\mathrm{mlU} / \mathrm{mL})$ \\
FSH $(\mathrm{mlU} / \mathrm{mL})$ \\
Oestradiol $(\mathrm{pg} / \mathrm{mL})$ \\
\hline
\end{tabular}

\begin{tabular}{ccc}
\hline $\begin{array}{c}\text { Reference } \\
\text { range }\end{array}$ & $\begin{array}{c}\text { Previous } \\
\text { hospitalisation }\end{array}$ \\
\cline { 1 - 1 } $0.50-4.30$ & & 1.101 \\
$2.30-4.10$ & & 4.97 \\
$0.70-1.70$ & & 2.6 \\
$<34$ & & \\
$0.13-9.88$ & \\
$80-216$ & \\
$6.1-30.5$ & \\
$7.2-63.3$ & \\
$7.1-19.6$ & \\
$5.72-64.31$ & \\
$<158$ & \\
$<47.0$ & \\
\hline
\end{tabular}

\begin{tabular}{c}
\hline $\begin{array}{c}\text { On } \\
\text { admission }\end{array}$ \\
\hline 0.886 \\
6.04 \\
2.75 \\
40 \\
0.03 \\
107 \\
6 \\
24.7 \\
8.4 \\
9.8 \\
37 \\
21 \\
\hline
\end{tabular}

\begin{tabular}{c}
$\begin{array}{c}\text { After } 3 \\
\text { days of } \\
\text { SSA }\end{array}$ \\
\hline 0.187 \\
5.38 \\
2.52 \\
\end{tabular}

\begin{tabular}{c}
\hline $\begin{array}{c}\mathbf{4} \text { days } \\
\text { post-TSS }\end{array}$ \\
\hline$<0.003$ \\
4.34 \\
2.28 \\
0.58 \\
102 \\
6 \\
25.5 \\
12.4 \\
8.8 \\
28.7 \\
17 \\
\hline
\end{tabular}

\begin{tabular}{c}
\hline 7 days \\
post-TSS \\
\hline$<0.003$ \\
4.81
\end{tabular}

\begin{tabular}{|c|c|}
\hline $\begin{array}{c}12 \text { days } \\
\text { post-TSS }\end{array}$ & $\begin{array}{l}\text { A month } \\
\text { post-TSS }\end{array}$ \\
\hline 0.125 & $<0.003$ \\
\hline 4.79 & 4.56 \\
\hline
\end{tabular}

The kits for the measurement of TSH, FT3 and FT4 are as follows. ARCHITECT (Abbott, Japan) at the previous hospital, and lumipulse Presto II (Fujirebio, Japan) after admission to our hospital.

FSH, follicle-stimulating hormone; fT3, free tri-iodothyronine; fT4, free thyroxine; GH, growth hormone; IGF1, insulin-like growth factor 1; LH, luteinising hormone; SSA, somatostatin analogue; TSH, thyroid-stimulating hormone; TSS, trans-sphenoidal surgery. 


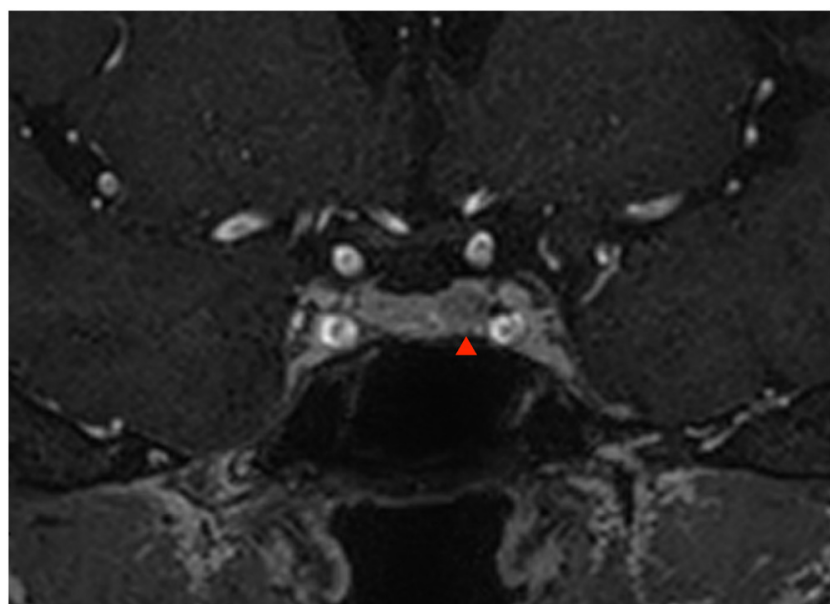

Figure 1

Enhanced T1-weighted coronal MRI showing a 5-mm tumour with poor contrast in the sella (arrow).

lower limit of the reference range when the TSHoma is completely removed.

Complications of destructive thyroiditis were suspected ${ }^{99} \mathrm{mTCO}_{4}$ scintigraphy was performed on postoperative day 12 . The scan showed accumulation in both normal thyroid glands and nodules, which was not compatible with destructive thyroiditis. Furthermore, 1 month later, suppression of serum TSH and elevated FT3 and FT4 levels persisted. Therefore, ${ }^{131}$ I scintigraphy was performed on postoperative day 27. Although we could not find a 'hot nodule' in thyroid scintigraphy before the operation (Fig. 2C), as shown by single-photon emission $\mathrm{CT} / \mathrm{CT}$, the area with strong signal shifted from the normal thyroid gland to the nodule, and the signal in the normal thyroid was diminished (Fig. 2D). This result led us to the diagnosis of AFTN. The patient was treated temporarily using methimazole (MMI), and radio-isotope treatment

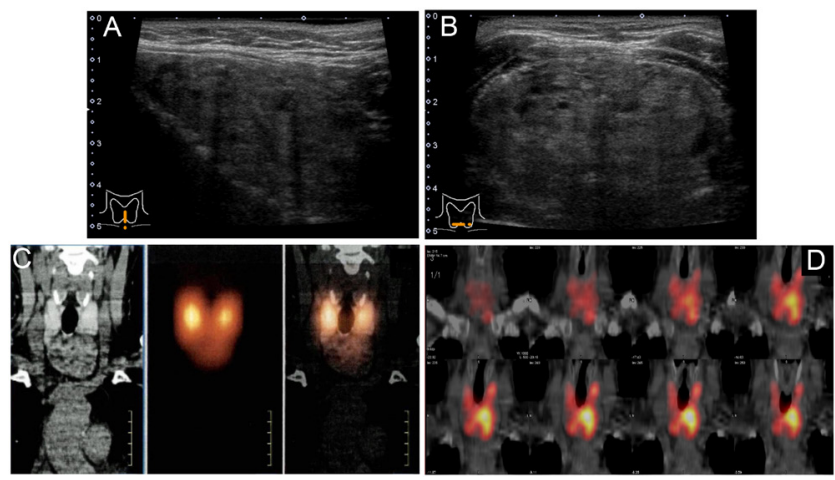

\section{Figure 2}

Images of thyroid nodule. (A and B), Ultrasound images; (C), Thyroid scintigraphy SPECT/CT figure before surgery for TSHoma $\left({ }^{99 m} \mathbf{T c O}_{4}\right)$; (D), Thyroid scintigraphy SPECT/CT figure after surgery for TSHoma (131I).

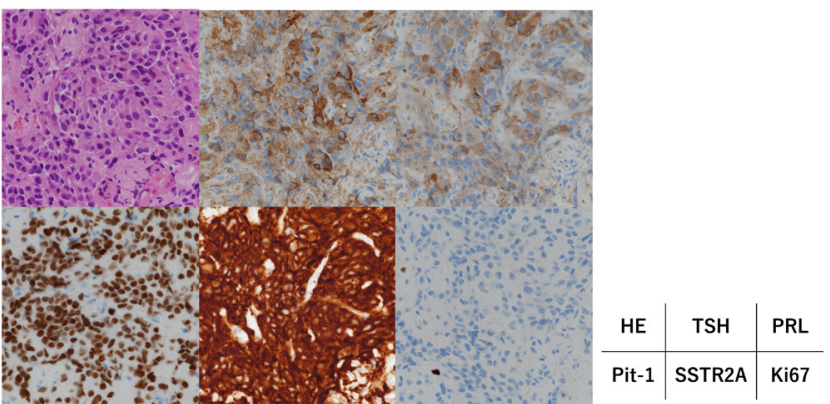

Figure 3

Pathological results. The tumour showed a monotonous cellular pattern and was TSH-positive, PRL-positive and PIT-1-positive, providing evidence that the tumour was a TSHoma. The results also demonstrated SSTR2 positivity, and the Ki-67 labelling index was $0.5 \%$. PIT-1, pituitary specific transcription factor-1; SSTR2, somatostatin receptor type 2.

was performed in accordance with her choice. The nodule has been cytologically confirmed to be benign.

\section{Discussion}

TSHoma can cause hyperthyroidism due to the autonomous secretion of TSH. The median serum TSH level upon TSHoma diagnosis is $3.08 \mu \mathrm{IU} / \mathrm{mL}$, while TSH levels only exceed the upper limit of the reference value in approximately $30 \%$ of TSHomas (2). Therefore, the key to diagnosing TSHoma is not the presence of a high serum TSH level but rather an inappropriately suppressed TSH level, that is, SITSH, which should not be missed. The first-line treatment for TSHoma is surgical removal of the tumour. It is also important to address thyrotoxicosis prior to surgery. Somatostatin analogues, such as octreotide and lanreotide, act on tumour-expressed SSTR2 and reduce hormone synthesis, thus shrinking the size of the tumour. Octreotide lowers serum TSH levels and normalises thyroid function in more than $80 \%$ of TSHomas (4).

In the present case, octreotide certainly lowered the serum TSH level, but not FT3 or FT4 levels, which indicated an atypical course. This course itself suggested the autonomous secretion of thyroid hormones by AFTNs, which we noted in hindsight. Furthermore, the preoperative thyroid scintigraphy in this case did not show any hot nodules that could be diagnosed as AFTNs. Normally, thyroid hormones secreted by AFTN cause negative feedback to the normal pituitary gland, resulting in suppressed hormone production by the normal thyroid gland. In our case, however, TSH was also autonomously secreted from the pituitary tumour, suggesting that hormone production in the normal thyroid gland was not suppressed. One month after surgery, when the serum TSH level was markedly reduced and the patient showed 
findings of primary hyperthyroidism, we performed repeat thyroid scintigraphy. The uptake of radioactive iodine in the normal thyroid gland was suppressed, and the thyroid nodules were depicted as hot nodules. The nodules were found to be functional only after surgery to remove a pituitary tumour.

At first, we speculated that painless thyroiditis might be the cause of the thyroid hormone changes after TSHoma surgery in this case because we had used potassium iodide preoperatively. Potassium iodide impairs the organisation of thyroid hormones and inhibits the synthesis of thyroid hormones; thus, it is frequently used in thyroid crisis and severe Graves' disease to rapidly stabilise thyroid function in Japan. However, there is a case report of Lugol's solutioninduced painless thyroiditis (5). While rare, we have also experienced iodine-induced thyrotoxicosis. Therefore, thyroid scintigraphy was performed on postoperative day 12. The results showed a negative result of painless thyroiditis. Taken together, the scintigraph images on day 12 captured the process of dynamic changes in thyroid function following the suppression of TSH. A limitation of this report, however, is that we used different radionuclides for the preoperative and postoperative thyroid scintigraphy. Therefore, we cannot exclude the possibility that the difference of nuclides might had affected the intensity of the accumulation.

Case reports of AFTN combined with TSHoma are rare, likely because TSHoma is an uncommon disease. The first report of this combination was published in 1994 (6). Although that report did not mention that the tumour was completely resected, the diagnosis of AFTN was made sometime after the TSH level declined with surgery. More recently, Aksoy et al. reported a woman diagnosed with toxic multinodular goitre (TMNG) 2 months after transsphenoidal surgery (TSS) for a TSH and growth hormone (GH)-secreting pituitary adenoma (GHoma), with a rapid decrease in TSH levels and the appearance of thyrotoxic symptoms (7). Similar to our case, this patient did not show uptake in the thyroid nodule on preoperative scintigraphy. Active somatic mutations in TSH receptors (TSHR) and GNAS have been identified in a large proportion of AFTNs. However, whole-genome analysis of TSHoma has not revealed mutations in the genes associated with $\mathrm{G}$ protein (8); thus, the involvement of genetic mutations shared by AFTNs and TSHoma is unknown.

On the other hand, approximately half of GHomas, which are induced to differentiate by the same transcription factor as TSHomas (PIT-1), have active GNAS mutations. Furthermore, McCune-Albright's syndrome, a mosaic mutation of GNAS, is associated with GHoma and AFTNs. In this patient, there were no physical findings of acromegaly, and immunostaining was negative for $\mathrm{GH}$ staining.

In this case, it was difficult to evaluate the function of the thyroid nodules preoperatively due to the development of autonomous hormone secretion in multiple organs: the pituitary and thyroid. While paradoxical, this case clearly illustrates how the diagnosis of AFTNs is dependent on TSH levels. Additionally, it is important to return to the basics and re-evaluate the diagnosis when facing a course of events not centrally explained.

\section{Declaration of interest}

The authors declare that there is no conflict of interest that could be perceived as prejudicing the impartiality of the research reported.

\section{Funding}

This research did not receive any specific grant from any funding agency in the public, commercial or not-for-profit sector.

\section{Patient consent}

Written signed consent has been obtained from the patient for publication of the submitted article.

\section{Author contribution statement}

Keita Tatsushima, Akira Takeshita and Shuji Fukata: Endocrinologists of the patient. Hiroshi Nishioka, Noriaki Fukuhara and Mitsuo Yamaguchi-Okada: Neurosurgeon of the patient. Yasuhiro Takeuchi: Endocrinologist (Chief) collaborate with the discussion. All authors have seen and approved the final manuscript for submission.

\section{References}

1 Amlashi FG \& Tritos NA. Thyrotropin-secreting pituitary adenomas: epidemiology, diagnosis, and management. Endocrine 201652 427-440. (https://doi.org/10.1007/s12020-016-0863-3)

2 Yamada S, Fukuhara N, Horiguchi K, Yamaguchi-Okada M, Nishioka H, Takeshita A, Takeuchi Y, Ito J \& Inoshita N. Clinicopathological characteristics and therapeutic outcomes in thyrotropin-secreting pituitary adenomas: a single-center study of 90 cases. Journal of Neurosurgery 2014121 1462-1473. (https://doi org/10.3171/2014.7.JNS1471)

3 Nishihara E, Amino N, Maekawa K, Yoshida H, Ito M, Kubota S, Fukata S \& Miyauchi A. Prevalence of TSH receptor and Gs $\alpha$ mutations in 45 autonomously functioning thyroid nodules in Japan. Endocrine Journal 200956 791-798. (https://doi.org/10.1507/ endocrj.k09e-073)

4 Fukuhara N, Horiguchi K, Nishioka H, Suzuki H, Takeshita A, Takeuchi Y, Inoshita N \& Yamada S. Short-term preoperative octreotide treatment for TSH-secreting pituitary adenoma. Endocrine Journal 201562 21-27. (https://doi.org/10.1507/endocrj.EJ14-0118) 
5 Yang JW \& How J. Lugol's solution-induced painless thyroiditis. Endocrinology, Diabetes and Metabolism Case Reports 2017 2017Article ID: 17-0034. (https://doi.org/10.1530/EDM-17-0034)

6 Abs R, Stevenaert A \& Beckers A. Autonomously functioning thyroid nodules in a patient with a thyrotropin-secreting pituitary adenoma: possible cause - effect relationship. European Journal of Endocrinology 1994131 355-358. (https://doi.org/10.1530/ eje.0.1310355)
7 Aksoy DY, Gedik A, Cinar N, Soylemezoglu F, Berker M \& Gurlek OA. Thyrotropinoma and multinodular goiter: a diagnostic challenge for hyperthyroidism. Journal of Research in Medical Sciences 201318 1008-1010.

8 Sapkota S, Horiguchi K, Tosaka M, Yamada S \& Yamada M. Wholeexome sequencing study of thyrotropin-secreting pituitary adenomas. Journal of Clinical Endocrinology and Metabolism 2017102 566-575. (https://doi.org/10.1210/jc.2016-2261)

Received in final form 21 February 2021

Accepted 6 April 2021 